\title{
Efecto de budesonida sobre la densidad y el metabolismo óseo en niños asmáticos
}

\author{
Mariela Paoli de Valeri, M.C., ${ }^{(1)}$ Elsy M. Gómez, M.C., ${ }^{(1)}$ Ezio Valeri, M.C., ${ }^{(2)}$
}

Rafael Salinas, M.C., (3) Gabriela A. Bellabarba, Lic. en Bioanálisis, M. Sc.(4)

\section{Paoli de Valeri M, Gómez EM, Valeri E, Salinas R, Bellabarba GA. Efecto de budesonida sobre la densidad y el metabolismo óseo en niños asmáticos. Salud Publica Mex 2000;42:309-314.}

\begin{abstract}
Resumen
Objetivo. Determinar el efecto de la administración de budesonida inhalado sobre la densidad, el contenido mineral y el metabolismo óseo en niños asmáticos. Material y métodos. Estudio transversal realizado de septiembre de 1996 a julio de 1997, en el que se estudiaron 38 niños y niñas de entre 6 y 11 años de edad, seleccionados de la consulta externa de neumonología infantil del Instituto Autónomo Hospital U niversitario de Los Andes, Mérida,Venezuela. Se constituyeron tres grupos: nueve niños asmáticos tratados con budesonida inhalado (300 $\mu$ g/día en promedio) durante más de seis meses, (grupo A), 14 niños asmáticos que no recibieron tratamiento esteroideo (grupo B) y 15 niños no asmáticos (grupo C). A todos se les practicó una evaluación ósea con marcadores de formación y resorción y con una medición de la densidad (DMO) y el contenido mineral óseo (CMO). Se calcularon medidas de tendencia central y de dispersión, se realizó análisis de varianza, y se utilizaron las pruebas de Fisher y Scheffé para comparación de medias. Resultados. En los grupos estudiados (A, B y C) los niveles séricos de calcio fueron $9.1 \pm 0.3 ; 9.6 \pm 0.4$; $9.3 \pm 0.6 \mathrm{mg} / \mathrm{ml} ;$ los de osteocalcina fueron $14.8 \pm 4.6$; $13.0 \pm 2.5 ; 11.9 \pm 3.4 \mathrm{ng} / \mathrm{dl}$; los de telo péptido carboxiterminal del colágeno tipo I (ICTP) fueron 19.6 $\pm 16.5 ; 14.2 \pm 15.4$; $13.0 \pm 18.3 \mu \mathrm{g} / \mathrm{l}$; la DMO fue de $0.67 \pm 0.06 ; 0.68 \pm 0.06$; $0.69 \pm 0.06 \mathrm{~g} / \mathrm{cm}^{2}$, y el CMO fue de $1158.8 \pm 217.4$; $1106.4 \pm 256.1 ; 1176.5 \pm 240.5$ g, respectivamente. No hubo diferencias significativas entre los grupos. Conclusiones.
\end{abstract}

Paoli de Valeri M, Gómez EM, Valeri E, Salinas R, Bellabarba GA.

Effect of budesonide on bone density and bone metabolism of asthmatic children. Salud Publica Mex 2000;42:309-314.

\begin{abstract}
A bstract
Objective. To assess the effect of inhaled budesonide on the mineral density, content and bone metabolism in children with asthma. Material and methods. From September 1996 to July 1997, a cross-sectional study was conducted in 38 prepubertal children aged 6 to 11 years, selected from the pediatric chest outpatient clinic of the Instituto Autónomo Hospital Universitario de Los Andes, Mérida, Venezuela. Three study groups were assembled: 9 asthmatic children treated with inhaled budesonide $(300 \mu \mathrm{g} / \mathrm{day})$ for over 6 months (Group A); 14 asthmatic children not treated with inhaled corticosteroids (Group B); and 15 nonasthmatic children ( $\mathrm{G}$ roup $\mathrm{C}$ ). All of them underwent testing of bone formation and resorption markers, and measurement of bone mineral density (DMO) and content (CMO). Statistical analysis consisted of central tendency and dispersion measures, analysis of variance, and Fisher and Scheffe tests for comparison of means. Results In the groups studied $(A, B$, and $C)$ calcium serum levels were 9.1 $1 \pm 0.3 ; 9.6 \pm 0.4$; $9.3 \pm 0.6 \mathrm{mg} / \mathrm{ml}$, respectively; osteocalcine levels were $14.8 \pm 4.6 ; 13.0 \pm 2.5 ; 11.9 \pm 3.4 \mathrm{ng} / \mathrm{dl}$; the type I collagen car-

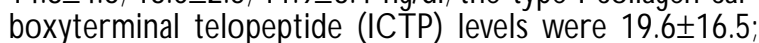
$14.2 \pm 15.4 ; 13.0 \pm 18.3 \mu \mathrm{g} / \mathrm{l}$; the DMO levels were $0.67 \pm 0.06$; $0.68 \pm 0.06 ; 0.69 \pm 0.06 \mathrm{~g} / \mathrm{cm}^{2}$; and the CMO levels were $1158.8 \pm 217.4 ; 1106.4 \pm 256.1 ; 1176.5 \pm 240.5$ g, respectively. No statistically significant differences were observed between the groups. Conclusions. The administration of 100-400 $\mu \mathrm{g} /$ day of inhaled budesonide for a period of six
\end{abstract}

(1) Unidad de Endocrinología, Universidad de Los Andes - Hospital Universitario de Los Andes (ULA-HULA). Mérida,Venezuela.

(2) Unidad de N eumonología, ULA-HULA. Mérida, Venezuela.

(3) Instituto de Medicina Nuclear, Universidad de Los Andes (ULA). MéridaVenezuela.

(4) Departamento de Fisiopatología, ULA, Mérida, Venezuela.

Fecha de recibido: 23 de junio de 1999 - Fecha de aprobado: 27 de julio de 2000

Solicitud de sobretiros: Gabriela A. Bellabarba. D epartamento de Fisiopatología. A partado postal 42. Mérida, Venezuela. Correo electrónico: arabella@ telcel.net.ve 
La administración de budesonida inhalada en dosis de 100 a $400 \mu \mathrm{g} /$ día por un periodo de seis meses no produce modificaciones en la densidad y en el metabolismo óseo en niños asmáticos.

Palabras claves: niño; asma; esteroides/administración por inhalación; densidad ósea;Venezuela months, did not change the bone mineral density and metabolism of asthmatic children.

Key words: child; asthma; steroids/administration, inhalation; bone density;Venezuela
E 1 uso de los esteroides inhalados para el tratamiento de asma ha ido en aumento en los últimos años, ya que minimizan la posibilidad de efectos colaterales en comparación con los esteroides sistémicos, pudiendo prevenir algunos de los cambios irreversibles que se producen a largo plazo sobre la función pulmonar. ${ }^{1-3}$ Los glucocorticoides sistémicos reducen la masa ósea, ${ }^{1,4,5}$ mientras que el efecto de los esteroides inhalados sobre el hueso es tema de controversia. ${ }^{4,6-8}$ Pocos estudios han investigado la densidad mineral y el metabolismo óseo en niños asmáticos que reciben esteroides inhalados, y los resultados han sido heterogéneos en función del rango de edad de los pacientes, dosis, tipo y duración de la terapia, y métodos de medida de la masa ósea. Algunos autores han informado disminución en la formación ósea, ${ }^{9-12}$ mientras que otros no encuentran diferencias ni en el metabolismo ni en la densidad ósea. ${ }^{13,14}$ Dada la alta frecuencia de asma en la población infantil y el uso cada vez más frecuente de esteroides inhalados para su control, se hace necesario definir la posible repercusión de estos medicamentos sobre la densidad y el metabolismo óseo. En consecuencia, se realizó este estudio con el objetivo de evaluar la densidad, el contenido mineral y el metabolismo óseo en niños asmáticos tratados con un glucocorticoide inhalado (budesonida), en comparación con niños asmáticos sin esteroides inhalados y con niños no asmáticos.

\section{Material y métodos}

En este estudio de corte transversal, se estudiaron 38 niños prepuberales, entre 6 y 11 años de edad, previo consentimiento de los representantes de su participación en esta investigación, de septiembre de 1996 a julio 1997. Fue seleccionado este rango de edad para evitar la influencia de las hormonas sexuales sobre el hueso en los diferentes estadíos puberales. De la consulta externa de neumonología infantil del Instituto Autónomo Hospital Universitario de Los Andes, Mérida, Venezuela, se seleccionaron 23 niños con asma leve intermitente, leve y moderada persistente, determinada por evaluación clínica y espirométrica. ${ }^{15,16}$ Los
15 niños restantes fueron no asmáticos seleccionados de la población general; se dividieron en tres grupos: grupo $\mathrm{A}$, nueve niños con asma leve o moderada persistente, tres hombres y seis mujeres, tratados con budesonida (Pulmicort-Novopharma ${ }^{\circledR}$ ) a una dosis de 100 a $400 \mu \mathrm{g} /$ día $(\bar{X}=300$ ug/día $)$. En el momento de iniciar el estudio todos los niños llevaban más de seis meses recibiendo el tratamiento $(7.4 \pm 1.2$ meses, rango de 6 a 12 meses). Seis de estos niños recibían broncodilatadores orales o inhalados como clembuterol, salbutamol o fenoterol de acuerdo con la necesidad de uso en función de los síntomas; grupo B, 14 niños con asma leve intermitente, siete niños y siete niñas sin tratamiento esteroideo. Nueve de estos pacientes eran tratados con salbutamol, uno con teofilina, tres con mezclas de teofilina y salbutamol, y el último recibía salbutamol, teofilina y cromoglicato sódico inhalado. Los integrantes de este grupo nunca recibieron tratamiento con esteroides inhalados; grupo C, 15 niños no asmáticos, ocho hombres y siete mujeres. Fueron excluidos los niños con asma severa, aquellos con ingesta de calcio menor a la requerida para su edad, ${ }^{17}$ los que padecían enfermedades que alteran la densidad o el metabolismo óseo y aquellos niños tratados con esteroides orales o parenterales durante los seis meses previos a la investigación o con medicamentos que pueden variar la densidad o el metabolismo óseo.

A todos los niños de cada grupo se les practicó:

- Examen físico que incluyó peso, talla y cálculo del indice de masa corporal $(\mathrm{IMC}=$ peso en $\mathrm{kg} /$ talla en $\mathrm{m}^{2}$ ).

- Rayos X de mano izquierda para la determinación de la edad ósea, la que fue evaluada por el mismo observador, utilizando el método de Greulich y Pyle.

- Extracción de una muestra de sangre en ayunas, a las 8 de la mañana, de la vena antecubital, con separación del suero por centrifugación y almacenamiento del mismo hasta su procesamiento. En el suero se cuantificaron los niveles de osteocalcina, como marcador de formación ósea, de ICTP (te- 
lopéptido carboxiterminal del colágeno tipo 1) como marcador de resorción ósea y calcio sérico total.

- Determinación de la relación calcio/creatinina parcial en una muestra de la segunda orina de la mañana.

- Exploración con absorciometría de rayos X de doble energía (DEXA) de cuerpo total para determinar el contenido mineral óseo, la densidad mineral ósea y la relación contenido mineral óseo/porcentaje de tejido graso libre.

La osteocalcina se determinó por ensayo inmunorradiométrico (IRMA) usando kits comerciales del Nichols Institute Diagnostics and Immutopics (San Juan Capistrano, California, Estados Unidos de América-EUA-). El coeficiente de variación intraensayo (CV) fue de $3 \%$ y el CV interensayo fue de $5 \%$. El ICTP se determinó por radioinmunoanálisis (RIA), utilizando kits comerciales de Incstar Corporation (Stillwater, Minnesota, EUA). Las biopipetas de precisión se calibraron periódicamente y nunca se reutilizaron las puntas. Las muestras de sangre se colocaron en tubos nuevos, se centrifugaron en frío, se fraccionaron en alícuotas para evitar congelar y descongelar la misma muestra, se almacenaron a $-70{ }^{\circ} \mathrm{C}$ y nunca se utilizaron muestras hemolisadas. El CV intraensayo fue de $4.3 \%$ y el CV interensayo fue de $5.5 \%$.

La creatinina urinaria se determinó por la metodología de Jaffé de acuerdo con el procedimiento de análisis de Ciba-Corning Diagnostic Corporation (Oberlin, Ohio, EUA). El calcio sérico y urinario se determinaron usando la metodología creosolfteleínocomplexona de acuerdo con el procedimiento de análisis de Ciba-Corning Diagnostic Corporation (Oberlin, Ohio, EUA). La exploración con DEXA se realizó con el empleo del sistema XR-37 de la Norland, Corporation con filtración dinámica. El equipo fue calibrado diariamente utilizando un fantoma de calibración de 77 pasos adicional a un fantoma de control de calidad de hidroxiapatita provisto por la casa fabricante. La precisión de las medidas aportadas en cuerpo total por este equipo varían entre 0.6 y $0.9 \%$ durante el tiempo de exploración del presente estudio con tiempo de exploración individual de entre 6 a 10 minutos. Todos los análisis fueron realizados por el mismo observador con el objeto de minimizar las variaciones interobservador. La variación dependiente del operador en la densidad mineral ósea $\left(\mathrm{DMO}, \mathrm{g} / \mathrm{cm}^{2}\right)$ y en el contenido mineral óseo $(\mathrm{CMO}$, g) en cuerpo total fue evaluada en 14 sujetos sanos, tres veces cada uno en un tiempo total de una semana, obteniéndose un coeficiente de variación de 1.03 y
$0.78 \%$, respectivamente. El operador del equipo Norland está certificado por la International Society for Clinical Densitometry.

Se calcularon medidas de tendencia central y de dispersión. La significancia estadística de las diferencias de los promedios entre los grupos se determinó mediante la aplicación del análisis de varianza (Anova) y pruebas de Fisher y Scheffé, ya que las variables estadísticas presentaron una distribución poblacional normal. Se consideró como significativo un valor de $p<0.05$.

\section{Resultados}

En el cuadro I se presenta la edad cronológica, la talla, el peso, el índice de masa corporal y la edad ósea de los niños asmáticos en tratamiento con budesonida (grupo A), de los niños asmáticos sin budesonida (grupo B) y de los niños no asmáticos (grupo C). Entre los grupos, los parámetros estudiados no fueron diferentes.

En el cuadro II se muestran los resultados de los niveles séricos de calcio, osteocalcina e ICTP y los datos urinarios de la relación calcio/creatinina. Esta última en los niños asmáticos tratados con budesonida fue similar a la del grupo de no asmáticos, pero significativamente mayor que la obtenida en los niños asmáticos sin budesonida. En las otras variables no hubo diferencias significativas entre los grupos, aunque se observa una tendencia hacia la elevación en los niveles de osteocalcina e ICTP en el grupo de niños asmáticos con budesonida, en comparación con los otros dos grupos.

\section{Cuadro I \\ Características físicas de los niños estudiados. \\ Instituto Autónomo Hospital Universitario de Los Andes, Mérida, Venezuela, SEPTIEMBRE DE 1996 A JULIO DE 1997}

\begin{tabular}{lrrr} 
& $\begin{array}{c}\text { Grupo } A \\
n=9 \\
X \pm D E\end{array}$ & $\begin{array}{c}\text { Grupo B } \\
n=14 \\
X \pm D E\end{array}$ & $\begin{array}{c}\text { Grupo } C \\
n=15 \\
X \pm D E\end{array}$ \\
& $8.4 \pm 1.7$ & $8.2 \pm 1.8$ & $8.5 \pm 1.5$ \\
Edad (años) & $131.4 \pm 8.4$ & $126.8 \pm 11.3$ & $128.6 \pm 8.3$ \\
\hline Talla $(\mathrm{cm})$ & $29.5 \pm 8.4$ & $26.0 \pm 6.7$ & $27.7 \pm 4.2$ \\
\hline Peso $(\mathrm{kg})$ & $16.7 \pm 2.6$ & $15.9 \pm 2.0$ & $16.8 \pm 1.3$ \\
\hline IMC $\left(\mathrm{kg} / \mathrm{m}^{2}\right)$ & $8.0 \pm 1.9$ & $7.7 \pm 1.8$ & $8.3 \pm 1.5$
\end{tabular}

$\bar{X}$ : media

DE: desviación estándar

IMC: indice de masa corporal

Grupo $A$ : niños asmáticos con budesonida

Grupo B: asmáticos sin budesonida

Grupo C: no asmáticos 


\section{Cuadro II \\ Características bioquímicas de los niños estudiados. Instituto Autónomo Hospital Universitario de Los Andes, Mérida, Venezuela, SEPTIEMBRE DE 1996 A JULIO DE 1997}

\begin{tabular}{|c|c|c|c|}
\hline & $\begin{array}{c}\text { Grupo A } \\
n=9 \\
\bar{X} \pm D E\end{array}$ & $\begin{array}{c}\text { Grupo B } \\
n=14 \\
\bar{X} \pm D E\end{array}$ & $\begin{array}{c}\text { Grupo C } \\
n=15 \\
\bar{X} \pm D E\end{array}$ \\
\hline Calcio (mg/ml) & $9.1 \pm 0.3$ & $9.6 \pm 0.4$ & $9.3 \pm 0.6$ \\
\hline O steocalcina (ng/dl) & $14.8 \pm 4.6$ & $13.0 \pm 2.5$ & $11.9 \pm 3.4$ \\
\hline ICTP (ug/l) & $19.6 \pm 16.5$ & $14.2 \pm 15.4$ & $13.0 \pm 18.3$ \\
\hline \multicolumn{4}{|c|}{$\begin{array}{l}\text { *p }=0.012 \text { vs. A y C } \\
\text { X: media } \\
\text { DE: desviación estándar } \\
\text { ICTP: telopéptido carboxiterminal del colágeno tipo } 1 \\
\text { Grupo A: niños asmáticos con budesonida } \\
\text { Grupo B: asmáticos sin budesonida } \\
\text { Grupo C: no asmáticos }\end{array}$} \\
\hline
\end{tabular}

En la figura 1 se presentan los niveles individuales de osteocalcina e ICTP en cada grupo estudiado. Se observa que los valores de osteocalcina son bastante homogéneos y sólo un paciente del grupo A tiene un valor mayor de $19 \mathrm{ng} / \mathrm{ml}$, el cual es el valor máximo del grupo control (grupo C). Por el contrario, en los niveles de ICTP se observa bastante dispersión de los resultados en todos los grupos; sin embargo, llama la atención que cinco (55.6\%) niños del grupo que recibió budesonida tienen niveles mayores de $15 \mathrm{ug} / \mathrm{l}$. En los otros grupos esta cifra es menos representativa, cinco pacientes $(35.7 \%)$ en el grupo de asmáticos sin budesonida y tres pacientes $(20 \%)$ en el grupo de niños no asmáticos. Cabe destacar que se usa este nivel de $15 \mu \mathrm{g} / 1$ de una manera arbitraria, ya que los niveles de referencia normales para ICTP en los niños no están establecidos y, además, los niveles utilizados por el kit comercial fueron determinados en pacientes adultos $(1.8-5 \mu \mathrm{g} / \mathrm{l})$.

En el cuadro III se muestran los resultados de la densidad mineral ósea (DMO), el contenido mineral óseo (CMO) y el contenido mineral óseo total/porcentaje de tejido graso libre (CMOt/\%TGL) en los grupos estudiados. No hubo diferencias estadísticamente significativas entre los grupos, aunque en el grupo de niños no asmáticos se obtuvo el valor más alto en la densidad y el contenido mineral óseo.

\section{Discusión}

En niños asmáticos la relación entre los cambios en los marcadores óseos y la administración de esteroi- des inhalados no está bien definida. ${ }^{18-20}$ Sorva y colaboradores ${ }^{21}$ informaron una disminución transitoria tanto en los marcadores de formación (osteocalcina y propéptido del procolágeno tipo 1- PICP) como en los de resorción ósea (ICTP y puentes cruzados de piridinolina urinario) y sugieren que la budesonida inhalado podría disminuir ligeramente el recambio óseo, pero sin cambios en la masa ósea. Otros autores ${ }^{13,22-24}$ han observado que la osteocalcina está reducida en los pacientes con asma bronquial independientemente del uso del esteroide inhalado. La observación de que la osteocalcina, sintetizada en el osteoblasto puede estimular la actividad del osteoclasto e inducir en parte la resorción y el recambio óseo, ${ }^{25,26}$ sugiere ésta que no puede considerarse sólo como un marcador de formación sino también de resorción ósea. En mujeres posmenopáusicas, con riesgo aumentado de fractura de cadera, se observan valores elevados de osteocalcina ${ }^{27}$ y un alto grado de correlación con el ICTP, ${ }_{1}^{10}$ que es un marcador de resorción ósea. En pacientes asmáticos tratados con esteroides inhalados se ha notificado una disminución de la formación ósea por la reducción del PICP. ${ }^{28}$ Kerstjens y colaboradore ${ }^{10}$ no comunican diferencias en este marcador, mientras que Crowley y colaboradores ${ }^{23}$ informan niveles elevados de PICP y sugieren que los glucocorticoides modifican el aclaramiento del mismo.

En este trabajo no observamos cambios significativos en el ICTP entre los niños asmáticos tratados con budesonida, los no tratados con esteroides y los no asmáticos; sin embargo, llama la atención que un porcentaje importante de los pacientes asmáticos que recibieron budesonida tuvieron niveles altos de ICTP; la importancia fisiológica de este hallazgo tendría que ser evaluada en estudios con un mayor número de pacientes y a largo plazo. En la literatura se han notificado niveles variables de ICTP en estos pacientes; algunos autores encuentran niveles disminuidos de ICTP en los niños asmáticos tratados con esteroides inhalados ${ }^{23}$ mientras que otros, ${ }^{10}$ no observaron aumento de ICTP.

Investigar la $\mathrm{DMO}$ en niños asmáticos que reciben esteroides inhalados es importante, considerando que la adquisición de un nivel óptimo de masa ósea durante la infancia y la adolescencia es esencial para prevenir una osteoporosis futura. ${ }^{29,30} \mathrm{En}$ los pacientes estudiados y tratados con budesonida inhalado no observamos cambios significativos en la densidad mineral ósea ni en el contenido mineral óseo. Este hallazgo, similar a lo informado por otros autores, $, 13,31-33$ es relevante, ya que el efecto de los esteroides inhalados sobre el hueso debe ser evaluado a largo plazo y con medidas específicas del estado óseo, como la den- 

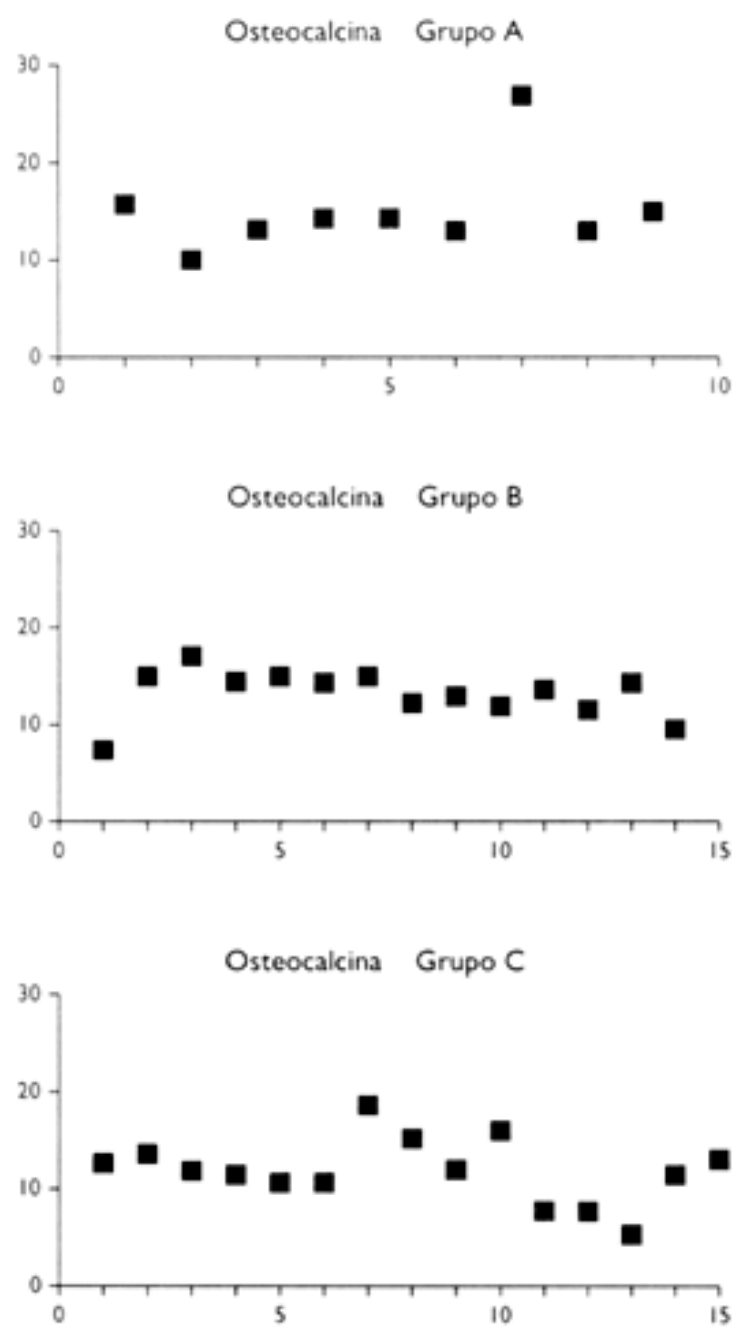
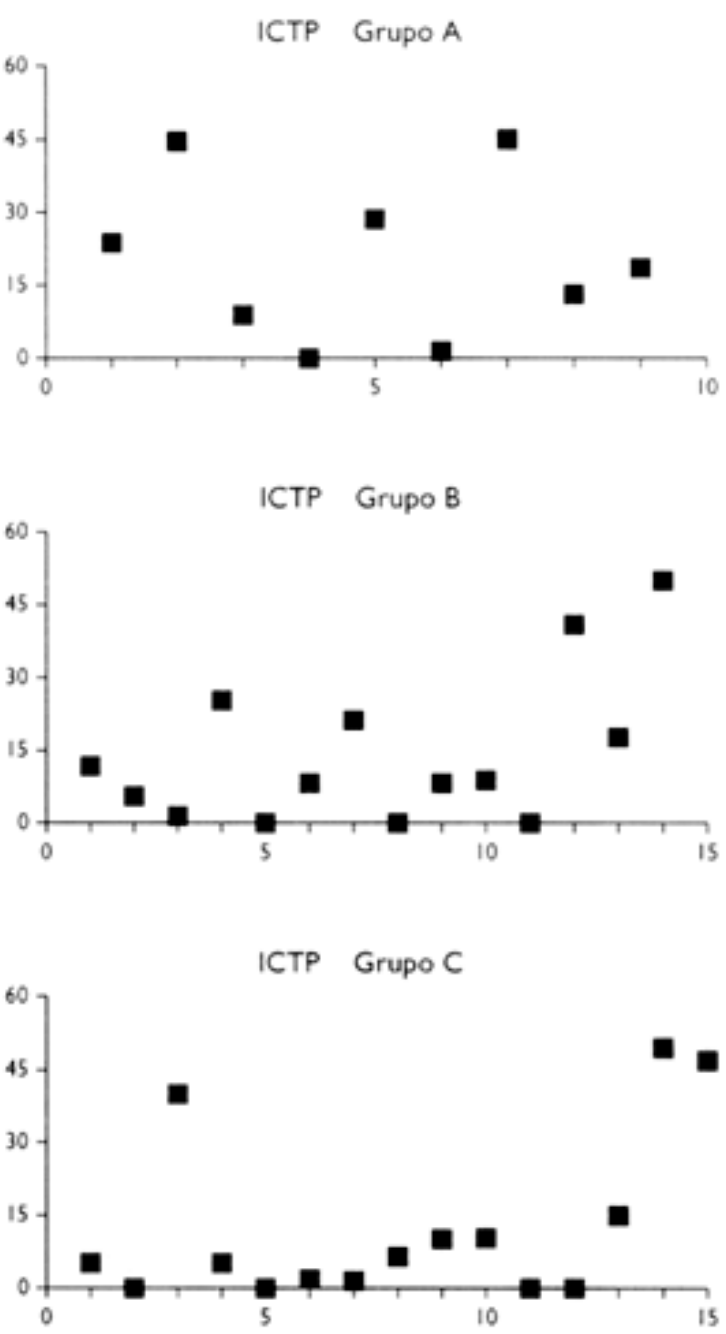

ICTP: telopéptido carboxiterminal del colágeno tipo 1

Figura 1. Niveles séricos individuales de osteocalcina (NG/DL) E ICTP ( $\mu$ G/L) en CADA GRUPO eStudiado. InStituto Autónomo Hospital Universitario de Los Andes, Mérida,Venezuela, de septiembre de 1996 a julio de 1997

sitometría ósea o el número de fracturas, más que con variaciones a corto plazo en los marcadores óseos. ${ }^{10,23}$ El estudio de Martinati y colaboradores ${ }^{31}$ incluyó la determinación de DMO cortical y trabecular (vertebral), al considerar que la mayor susceptibilidad a la pérdida ósea inducida por los glucocorticoides podría resultar más ostensible en aquellas áreas donde predominara el tipo de hueso trabecular; sin embargo, no hubo diferencias al respecto. Pacientes con asma severa que reciben esteroides inhalados a muy altas dosis y por tiempo prolongado podrían tener un riesgo de cierta pérdida ósea, pero esto es preferible a la alternativa de mantener un tratamiento con glucocorticoides orales para controlar la inflamación bronquial, con los que la pérdida ósea sería mucho mayor. ${ }^{18}$

Los resultados obtenidos en los niños asmáticos tratados con budesonida comparados tanto con los de niños asmáticos sin el esteroide como con los de niños no asmáticos de edad similar demuestran que en los niños asmáticos tratados con budesonida inhalado no hubo hipocalcemia ni diferencias en la masa ósea ni en los marcadores de formación y re- 


\section{Cuadro III \\ Marcadores óseos de LoS niÑos estudiados. Instituto Autónomo Hospital Universitario de Los Andes, Mérida, Venezuela, SEPTIEMBRE DE 1996 A JULIO DE 1997}

$\begin{array}{ccc}\text { Grupo } A & \text { Grupo } B & \text { Grupo } C \\ n=9 & n=14 & n=15 \\ \bar{X} \pm D E & \bar{X} \pm D E & \bar{X} \pm D E\end{array}$

\begin{tabular}{lcccccc} 
DMO $\left(\mathrm{g} / \mathrm{cm}^{2}\right)$ & $0.67 \pm$ & 0.06 & $0.68 \pm$ & 0.06 & $0.69 \pm$ & 0.06 \\
\hline CM0 $(\mathrm{g})$ & $1158.8 \pm 217.4$ & $1106.4 \pm 256.1$ & $1176.5 \pm 240.5$ \\
\hline CMO t/\%TGL & $6.9 \pm$ & 1.2 & $6.8 \pm$ & 0.9 & $6.6 \pm$ & 0.9
\end{tabular}

$\bar{X}$ : media

DE: desviación estándar

DMO : densidad mineral ósea

CMO : contenido mineral óseo

CMO t/\%TGL: relación contenido mineral óseo total/porcentaje de tejido graso libre

Grupo A: niños asmáticos con budesonida

Grupo B: asmáticos sin budesonida

Grupo $C$ : no asmáticos

sorción ósea; por ello, se concluye que la budesonida utilizada a dosis menores de 400 ug/día es una alternativa terapéutica sin efectos deletéreos sobre el hueso y que mejora las condiciones generales del niño asmático.

\section{Referencias}

1. Barnes P. Inhaled glucocorticoids for asthma. N Engl J Med 1995;332: 868-875

2. Calpin C, MacArthur C, Stephens D, Feldman W, Parkin P. Effectiveness of prophilactic inhaled steroids in childhood asthma:A systematic review of the literature. J Allergy C lin Immunol 1997;100:452-457.

3. D onahue J, W eiss S, Livingston J, Goetsch M, G reineder D, Platt R. Inhaled steroids and the risk of hospitalization for asthma. JAMA 1997; 277:887-891.

4. Volovitz B, Jacob A, Malik H, Kauschansky A, Varsano 1. Growth and pituitary adrenal function in children with severe asthma treated with inhaled budesonide. N Engl J Med 1993;329:1703-1708.

5. W ard MJ. Inhaled corticosteroids effect on bone? Respir Med 1993; 87supplA:33-36.

6. Hanania N, Chapman K, Kesten S. Adverse effects of inhaled corticosteroids. Am J Med 1995;98:196-208.

7. Gennari C. Glucocorticoid induced osteoporosis. Clin Endocrinol 1994:41:273-274.

8. Thomas B, Stanhope R, Grant D. Impaired growth in children with asthma during treatment with conventional doses of inhaled corticosteroids. Acta Pædiatr 1994:83:196-199.

9. Sorva $R$, Turpeinen M, Juntunen-Backman K, Karone SL, Sorva A. Effect of inhaled budesonide on serum markers of bone metabolism in children with asthma. J Allergy C lin Immunol 1992;90:808-815.

10. Kerstjens H, Postma D, van D oormaal J, van Zanten A, Brand P, D ekhuijzen $\mathrm{H}$ et al. Effects of short term and long term treatment with inhaled corticosteroids on bone metabolism in patients with airways obstruction. Thorax 1994;49:652-656.

11. Toogood JH, Jennings B, H odsman A, Baskerville J, Fraber L. Effects of dose and dosing schedule of in haled budesonide on bone turnover. J Allergy C lin Immunol 1991;88:572-580.

12. Morrison D, Routledge $P, C$ apewell $S$. Bone turnover during short course prednisolone treatment in patients with chronic obstructive airways disease. Thorax 1992;47:418-420.

13. König P, Hillman L, C evantes C, Levine C, Maloney C, D ouglass B et al. Bone metabolism in children with asthma treated with inhaled beclomethasone dipropionate. J Pediatr 1993;122:219-226.

14. Matsumoto $H$, Ishihara K, Hasegawa T, Sakamoto H, U meda B. Effects on bone metabolism of asthma treatment with beclomethasone dipropionate (BDP) inhalation and short term burst of oral steroids. $N$ ippon Kyobu Shikkan Gakkai Zasshi 1994:32:970-976.

15. Joint task fork of the A cademy and the American College of Allergy and Immunology. C lassification of asthma severity. J Allergy C lin Immunol 1995:96:749-751.

16. Manual de espirometría Fukuda-Sanyo. SP 200. Escala de obstrucción bronquial. Tokio 1986

17. $N$ ational Institute of Health. Consensus development panel on optimal calcium intake. JAMA 1994;272:1942-1948.

18. Woodcock A. Effects of inhaled corticosteroids on bone density and metabolism. J Allergy C lin Immunol 1998;101:S456-S459.

19. Pouw E, Prummel M, $O$ osting $H$, Roos C, Endert E. Beclomethasone inhalation decreases serum osteocalcin concentrations. BMJ 1991;302: 627-628.

20. Price J. Inhaled corticosteroids: C linical relevance of safety measures. Pediatr Pulmonol 1997;Suppl 15:40-45.

21. Sorva R,Tahtela R,Turpeinen M, Juntunen-Backman K, H aatelaT, Risteli $L$ et al. Changes in bone markers in children with asthma during inhaled budesonide and nedocromil treatments. A cta Paediatr 1996:85:1176-1180 22. D oull I, Freezer N, Holgate S. O steocalcin, growth, and inhaled corticosteroids: A prospective study. Arch D is Child 1996;74:497-501.

23. Crowley S, Trivedi P, Risteli L, Risteli J, Hindmarsh P, Brook C. Collagen metabolism and growth in prepuberal children with inhaled steroids. Pediatr 1998:132:409-413.

24. Chen-Kuang $\mathrm{N}$, Song-Chei $\mathrm{H}$, Chung-Bin H. Effect of short-course budesonide on the bone turnover of asthmatic children. Pediatr Pulmonol 1998:26:290-294.

25. G lowacki J, Lian J. Impaired recruitment and differentiation of osteoclast progenitors by osteocalcin-deplete bone implants. Cell Differ 1987;21:247-254

26. Lian JB, Tassinari M, G lowacki J. Resorption of implanted bone prepared from normal and warfarin-treated rats. J Clin Invest 1984;73: 1223-1226.

27. Szulc $P, C$ hapuy M, Meunier P, D elmas P. Serum undecarboxylated osteocalcin is a marker of the risk of hip fracture in ederly women. J Clin Invest 1993:11:189-192.

28. Ali N, Capewell S, W ard M. Bone turnover during high dose inhaled corticosteroid treatment. Thorax 1991;46:160-164

29. Bonjour J, Theintz G, Buchs B, Slosman D, Rizzoli R. Critical years and stages of puberty for spinal and femoral bone mass accumulation during adolescence.J Clin Endocrinol Metab 1991;73:555-563.

30.Carrié $\mathrm{A}$, Bonjour 1.0 steoporosis as a pediatric problem. Pediatr $\mathrm{Clin}$ N orth Am 1995;42:811-824.

31.Martinati L, Bertoldo F, Gasperi E, Micelli S, Boner A. Effect on cortical and trabecular bone mass of different anti-inflamatory treatments in preadolescent children with chronic asthma.Am J Respir C rit C are Med 1996; 153:232-236

32.Kinberg K, Hopp R, D O, Biven R, Gallagher J. Bone mineral density in normal and asthmatic children. J Allergy C lin Immunol 1994;94:490-497. 33.Efthimiou J, Barnes P. Effect of inhaled corticosteroids on bone and growth. Eur Respir J 1998;11:1167-1177. 